\title{
Amifostine protects from the peripheral sensory neuropathy induced by oxaliplatin in mice
}

\author{
A.F. Pereira $\mathbb{1}^{1 *}$, J.A. Lino $\mathbb{1}^{2 *}$, B.W.F. Alves $\mathbb{1}^{3}$, M.R.P. Lisboa $\mathbb{1}^{3}$, R.B. Pontes $\mathbb{1}^{4}$, \\ C.A.V.G. Leite $\mathbb{i}^{1}{ }^{1}$, R.B. Nogueira $\mathbb{i l}^{1}$, R.C.P. Lima-Júnior $\mathbb{i}^{1 \#}$, and M.L. Vale $\mathbb{B}^{1,3 \#}$ \\ ${ }^{1}$ Departamento de Fisiologia e Farmacologia, Faculdade de Medicina, Universidade Federal do Ceará, Fortaleza, CE, Brasil \\ ${ }^{2}$ Departamento de Medicina Clínica, Faculdade de Medicina, Universidade Federal do Ceará, Fortaleza, CE, Brasil \\ ${ }^{3}$ Departamento de Morfologia, Faculdade de Medicina, Universidade Federal do Ceará, Fortaleza, CE, Brasil \\ ${ }^{4}$ Departamento de Fisioterapia, Faculdade de Medicina, Universidade Federal do Ceará, Fortaleza, CE, Brasil
}

\begin{abstract}
Sensory neuropathy is a dose-limiting side effect of oxaliplatin-based cancer treatment. This study investigated the antinociceptive effect of amifostine and its potential neuroprotective mechanisms on the oxaliplatin-related peripheral sensory neuropathy in mice. Oxaliplatin $(1 \mathrm{mg} / \mathrm{kg}$ ) was injected intravenously in Swiss albino male mice twice a week (total of nine injections), while amifostine $(1,5,25,50$, and $100 \mathrm{mg} / \mathrm{kg}$ ) was administered subcutaneously 30 min before oxaliplatin. Mechanical and thermal nociceptive tests were performed once a week for 49 days. Additionally, c-Fos, nitrotyrosine, and activating transcription factor 3 (ATF3) immunoexpressions were assessed in the dorsal root ganglia. In all doses, amifostine prevented the development of mechanical hyperalgesia and thermal allodynia induced by oxaliplatin $(P<0.05)$. Amifostine at the dose of $25 \mathrm{mg} / \mathrm{kg}$ provided the best protection $(P<0.05)$. Moreover, amifostine protected against neuronal hyperactivation, nitrosative stress, and neuronal damage in the dorsal root ganglia, detected by the reduced expression of c-Fos, nitrotyrosine, and ATF3 ( $P<0.05$ vs the oxaliplatin-treated group). In conclusion, amifostine reduced the nociception induced by oxaliplatin in mice, suggesting the possible use of amifostine for the management of oxaliplatin-induced peripheral sensory neuropathy.
\end{abstract}

Key words: Oxaliplatin; Amifostine; Neuropathy; Chemotherapy; Pain

\section{Introduction}

Oxaliplatin is a third-generation platinum compound commonly used for the treatment of metastatic colon cancer (1). Despite significantly increasing patients' survival $(2,3)$, oxaliplatin causes several side effects that reduce their quality of life. The main side effect is neurotoxicity, manifested as peripheral sensory neuropathy (4) accompanied by significant neuronal damage in sensory nerves (5). Additionally, long-term treatment may affect proprioception, causing motor impairment (1). Symptoms of neuropathy, such as tingling, numbness in hands and feet, and pain, are common in patients treated with oxaliplatin, which may persist for more than five years (6). Hospitalization and dose reduction are common consequences of these side effects (7), which also cause treatment discontinuation (8). The management of peripheral sensory neuropathy symptoms includes antidepressants, anticonvulsants, opioids, and local anesthetics, among other drugs (9). However, there is no fully effective drug in preventing or reducing these symptoms, or even therapy capable of modifying the development of such toxicity.

Amifostine is a broad-spectrum cytoprotective agent that selectively protects non-tumor tissues from the toxic effects (10), such as nephrotoxicity (11) and neurotoxicity (12), induced by several chemotherapeutic regimens (13). Amifostine demonstrates a potent analgesic and antiinflammatory activity in experimental models of inflammation (14). However, there is limited and controversial clinical evidence of the effects of this drug on platinum compounds-related neuropathy $(15,16)$.

Therefore, this study investigated the antinociceptive effect of amifostine and the possible neuroprotective mechanism on the experimental peripheral sensory neuropathy induced by oxaliplatin in mice.

Correspondence: R.C.P. Lima-Júnior: < robertocesar@ufc.br > | M.L. Vale: <marianavale@ufc.br>

*These authors contributed equally to this work.

\#These authors have equal responsibility for this work. 


\section{Material and Methods}

\section{Animals}

The experiments were performed using Swiss albino male mice (Mus musculus), weighing 25-30 g, obtained from the Animal Facility of the Federal University of Ceará (Brazil). The animals were randomly divided into seven experimental groups $(n=6)$ and accommodated in appropriate cages with controlled temperature $\left(22 \pm 2^{\circ} \mathrm{C}\right)$ and light/dark cycle $(12 / 12 \mathrm{~h})$, receiving solid food and water ad libitum. The experiments were approved by the Ethics Committee in Animal Research of the Federal University of Ceará (protocol 27/08) and followed the local guidelines on the welfare of experimental animals.

\section{Induction of the peripheral sensory neuropathy}

According to Azevedo et al. (17), oxaliplatin (SigmaAldrich ${ }^{\circledR}$, USA), diluted in a $5 \%$ glucose solution (Dinâmica Química Contemporânea Ltda., Brazil), was administrated intravenously $(1 \mathrm{mg} / \mathrm{kg}$, twice a week, with a total of nine injections) in the lateral vein of the mice's tail. In the vehicle control group, a $5 \%$ glucose solution was administered intravenously. Mechanical and thermal nociceptive tests were conducted up to the 49th experimental day to evaluate the long-term effects of the oxaliplatin injections.

\section{Evaluation of the protective effect of amifostine on the oxaliplatin-induced peripheral sensory neuropathy}

Amifostine (Sigma-Aldrich ${ }^{\circledR}$ ) was dissolved in sterile saline solution and administered at the doses of 1, 5, 25, 50, or $100 \mathrm{mg} / \mathrm{kg}$, twice a week (total of nine injections) by the subcutaneous route $30 \mathrm{~min}$ before the injection of oxaliplatin (18). The oxaliplatin-treated group received the same volume of sterile saline solution. Mechanical and thermal nociceptive tests were performed to evaluate the protective effect of amifostine up to the 49th experimental day.

\section{Behavioral tests}

Plantar mechanical hyperalgesia test. The electronic von Frey apparatus (Insight ${ }^{\mathbb{R}}$, Brazil) was used to evaluate the intensity of plantar mechanical hyperalgesia of each animal. This equipment records the pressure in grams required to provoke a hind paw flexion followed by a flinch, through a mechanical stimulus of the paw by the tip of a rigid filament. The animals were housed in acrylic boxes $(12 \times 10 \times 17 \mathrm{~cm})$ under a wire grid $10 \mathrm{~min}$ before the behavioral test. A trained researcher, blind to the treatments, evaluated the mechanical hyperalgesia once a week before and after the drug injections $(17,19)$.

Cold allodynia tail immersion test. The tail immersion test was performed to evaluate the cold allodynia induced by a non-noxious temperature of $10^{\circ} \mathrm{C}(20,21)$. The total duration of the tail immersion was recorded in seconds, weekly, with a cut-off time of $120 \mathrm{~s}$ by a blind experimenter. One week before the experiment, the animals were adapted to the test.
Immunofluorescence. On the 28th experimental day, the dorsal root ganglia (DRG) were harvested for immunofluorescence assay. The mice were deeply anesthetized with an intraperitoneal administration of ketamine (100 mg/kg) (König do Brasil Ltda, Brazil) and xylazine (10 $\mathrm{mg} / \mathrm{kg}$ ) (König do Brasil Ltda). The anesthesia procedure was required considering the intracardiac perfusion that preceded the euthanasia method used in this study for the fixation of internal organs. The animals were intracardially perfused using $40 \mathrm{~mL}$ of sterile saline followed by $40 \mathrm{~mL}$ of a 4\% paraformaldehyde (PFA) (Sigma-Aldrich ${ }^{\mathbb{R}}$ ) solution. The DRG were then immersed in 4\% PFA for $2 \mathrm{~h}$ followed by cryoprotection with a $30 \%$ sucrose solution for two days. After this, the tissues were inserted in the TissueTek O.C.T. compound (Sakura ${ }^{\circledR}$, The Netherlands) and stored at a temperature of $-80^{\circ} \mathrm{C}$. Serial sections of the frozen DRG samples were acquired (10- $\mu \mathrm{m}$ thick) with a cryostat (Leica CM1850, Leica, Germany).

For the immunofluorescence assay, sample sections were fixed in methanol (Vetec Química Fina Ltda, Brazil), followed by antigenic recovery in $0.1 \mathrm{M}(\mathrm{pH} \mathrm{6.0)}$ citrate buffer, at a temperature of $95^{\circ} \mathrm{C}$. To allow the permeabilization of the nuclear membrane, $0.1 \%$ Triton X-100 (Sigma-Aldrich ${ }^{\circledR}$ ) was used. Then, a solution containing $5 \%$ bovine serum albumin (Sigma-Aldrich ${ }^{\mathbb{R}}$ ) added to 0.3 $\mathrm{M}$ glycine (Sigma-Aldrich ${ }^{\circledR}$ ) was used to block the nonspecific antibody binding. The incubation of the primary antibodies was carried out overnight with rabbit anti-c-Fos (Santa Cruz Biotechnology ${ }^{\circledR}$, USA), in a 1:200 dilution, rabbit anti-nitrotyrosine (Merck Millipore ${ }^{\mathbb{R}}$, USA), in a $1: 300$ dilution, and rabbit anti-activating transcription factor 3 (ATF3) $\left(\right.$ Abcam $^{\mathbb{R}}$, UK), in a 1:300 dilution. After this, the sections were incubated with the secondary antibody goat anti-rabbit IgG coupled to Alexa Fluor 568 (Invitrogen ${ }^{\circledR}$, Life Technologies, Thermo Fisher Scientific, USA), at a dilution of 1:400. The NeuN antibody conjugated with Alexa Fluor 488 (Merck Millipore ${ }^{\mathbb{R}}$ ) was used to label the neuronal cell bodies, at a dilution of 1:150. Slides were mounted with ProLong Gold Antifade Mountant (Invitrogen ${ }^{\circledR}$, Life Technologies, Thermo Fisher Scientific). The slides were photographed using a laser scanning confocal microscope (Zeiss LSM710, Carl Zeiss, Germany). The photographs were carefully taken with the same master gain and digital offset for all the samples, providing standardization for posterior analysis.

With the photomicrographs, the fluorescent area was quantified using an image analysis software (Fiji ImageJ, National Institutes of Health, USA). This quantification was blindly performed through the differentiation of the fluorescent pixels by the higher color saturation associated with the fluorescence (green or red). The color threshold previously set the higher and lower limits to define the selected and unselected pixels. The results obtained with the quantification of the fluorescent area were calculated by the positive fluorescent area concerning the fluorescence of NeuN and are reported in percentage. 


\section{Statistical analysis}

The data are reported as means \pm SE. The statistical differences among the experimental groups in the behavioral tests were assessed by two-way analysis of variance (ANOVA) followed by Tukey's post-test. To evaluate the other results, one-way ANOVA followed by Tukey's post-test was used. The level of significance was considered to be $\mathrm{P}<0.05$. GraphPad Prism version 6.00 for Windows (GraphPad Software, USA) was used for the analyses.

\section{Results}

Effect of amifostine on the mechanical nociceptive threshold in mice submitted to oxaliplatin-induced peripheral sensory neuropathy

The injection of oxaliplatin increased the variation of the paw withdrawal threshold significantly from the 21 st to the 49th day $(P<0.05)$ compared with the vehicle group (Figure 1A). Subcutaneous administration of amifostine, at doses of $1,5,25,50$, and $100 \mathrm{mg} / \mathrm{kg}$, prevented this increase of the variation from the 21 st day until the 49th day $(P<0.05)$, compared to the group treated with oxaliplatin (Figure 1A).

\section{Effect of amifostine on cold allodynia in mice} submitted to oxaliplatin-induced peripheral sensory neuropathy

Oxaliplatin significantly reduced the tail withdrawal time from the 14th to the 49th day $(P<0.05)$ compared with the group that received only the vehicle (Figure 1B). The subcutaneous injection of amifostine at doses of 1, 5, and $50 \mathrm{mg} / \mathrm{kg}$ prevented the decrease of tail withdrawal time from the 28th day until the 49th day $(P<0.05)$, while the doses of $100 \mathrm{mg} / \mathrm{kg}$ prevented this from the $21 \mathrm{st}$ to the 49th day $(\mathrm{P}<0.05)$, compared to the oxaliplatin group (Figure 1B). Only the dose of $25 \mathrm{mg} / \mathrm{kg}$ was able to prevent this reduction from the 14th day until the 49th day $(P<0.05)$ compared with the oxaliplatin group (Figure 1B). Thus, based on the behavioral results and total area under the curve (data not shown), the dose of $25 \mathrm{mg} / \mathrm{kg}$ was used for further analyses.

\section{Effect of amifostine on c-Fos expression in dorsal root ganglia}

The c-Fos immunofluorescence analysis is commonly used to demonstrate increased neuronal activity related to nociceptive behavior (22). Our results showed that oxaliplatin significantly increased $c$-Fos expression $(P<0.05)$ in neuronal cells (labeled with NeuN) from the DRG of the mice compared with the vehicle group. Amifostine $(25 \mathrm{mg} /$ $\mathrm{kg}$ ) treatment prevented the c-Fos increase $(P<0.05)$ in neuronal cells compared to the oxaliplatin group (Figure $2 \mathrm{~A}$ and Figure 3).

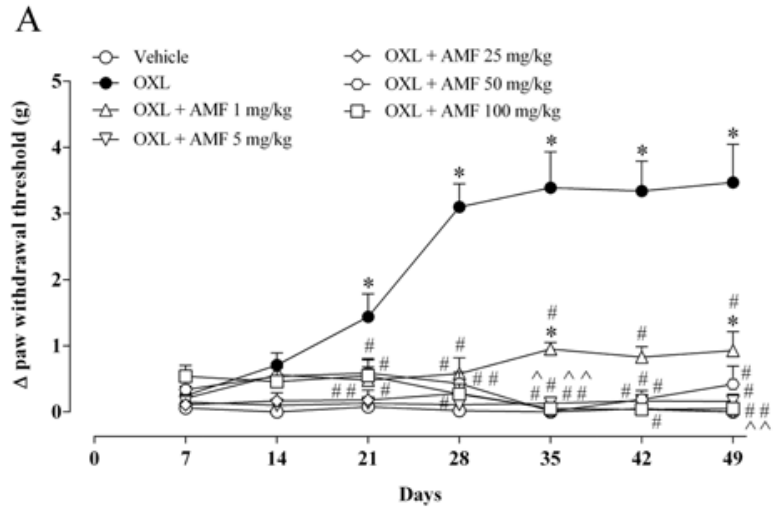

B

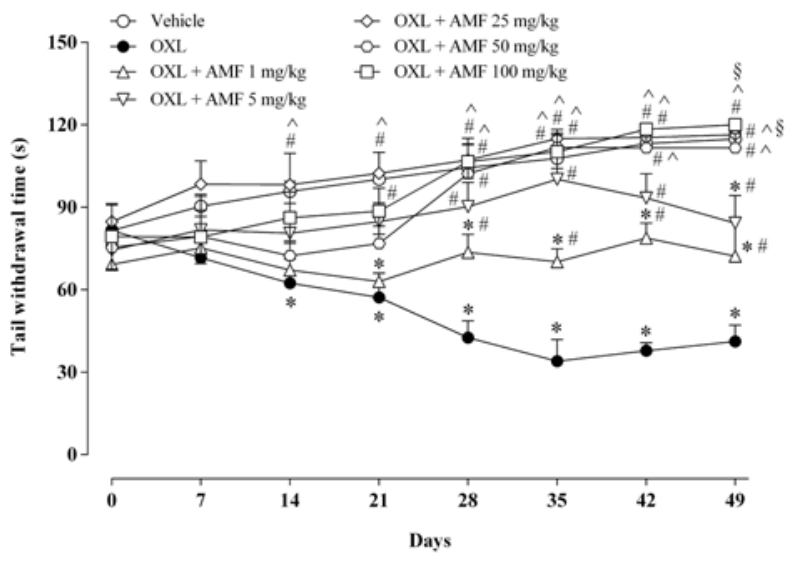

Figure 1. Effect of amifostine on behavioral tests. Oxaliplatin (OXL) $(1 \mathrm{mg} / \mathrm{kg})$ or $5 \%$ glucose (vehicle group) were injected intravenously, twice a week, for five weeks (a total of nine injections). Amifostine (AMF) $(1,5,25,50$, or $100 \mathrm{mg} / \mathrm{kg}$ ) was administered subcutaneously $30 \mathrm{~min}$ before the oxaliplatin injection. Von Frey electronic test (mechanical hyperalgesia) (A) and tail immersion test in cold water $\left(10^{\circ} \mathrm{C}\right)$ (cold allodynia) (B) were performed once a week, for 49 days. The results are reported as means \pm SE. ${ }^{*} \mathrm{P}<0.05$ vs vehicle; ${ }^{\#} \mathrm{P}<0.05$ vs $\mathrm{OXL}$; ${ }^{\wedge} \mathrm{P}<0.05 \mathrm{vs}$ OXL + AMF $1 \mathrm{mg} / \mathrm{kg} ;{ }^{\circledR} \mathrm{P}<0.05 \mathrm{vs} \mathrm{OXL}+\mathrm{AMF} 5 \mathrm{mg} / \mathrm{kg}$ (two-way ANOVA followed by Tukey post-test).

\section{Effect of amifostine on nitrotyrosine expression in dorsal root ganglia}

Nitrotyrosine immunoexpression was evaluated to investigate a possible anti-nitrosative mechanism involving the amifostine neuroprotective effect. In the oxaliplatintreated group, there was a significant increase of nitrotyrosine expression $(P<0.05)$ in neuronal cells (labeled with NeuN) of the DRG compared to the vehicle group. The treatment with amifostine prevented the increase of the nitrotyrosine expression $(P<0.05)$ in neuronal cells induced by the oxaliplatin injections compared with the oxaliplatin-treated group (Figure 2B and Figure 4). 


\section{Effect of amifostine on ATF3 expression in dorsal root} ganglia

ATF3 is a transcription factor expressed by neuronal cells and a known marker of neuronal injury and repair $(23,24)$. In the oxaliplatin group, a considerable increase
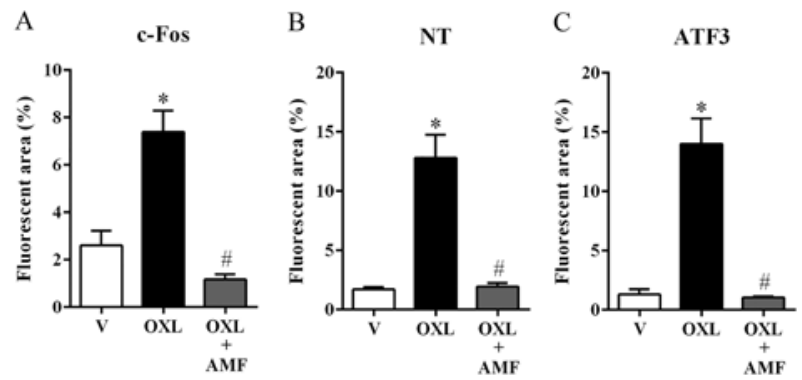

Figure 2. Fluorescent area quantification of c-Fos, nitrotyrosine (NT), and activating transcription factor 3 (ATF3) expression in the dorsal root ganglia of mice. The data are reported as means \pm SE of the percentage of positive fluorescent areas of c-Fos (A), NT (B), and ATF3 (C) expression concerning NeuN (neuronal marker) expression on the 28th experimental day. ${ }^{*} \mathrm{P}<0.05$ vs vehicle group, ${ }^{\#} \mathrm{P}<0.05$ vs $\mathrm{OXL}$ group (one-way ANOVA followed by Tukey post-test). AMF: amifostine; OXL: oxaliplatin; V: vehicle. in the ATF3 expression $(P<0.05)$ was found in neuronal cells (labeled with NeuN) of the DRG compared with the vehicle. Amifostine prevented this increase $(P<0.05)$ in neuronal cells, compared to the oxaliplatin group (Figure 2C and Figure 5).

\section{Discussion}

In the present study, the protective effect of amifostine was demonstrated in a model of peripheral sensory neuropathy induced by oxaliplatin in mice. This protection was confirmed by the modulation of neuropathy markers, such as c-Fos, nitrotyrosine, and ATF3.

Nociceptive behavioral tests were employed to confirm the development of neuropathy $(17,21)$. As expected, oxaliplatin induced a nociceptive response detected by the mechanical and thermal nociceptive tests. Several mechanisms, including oxidative (26) and nitrosative stresses $(17,27)$, contribute to the development of neuropathic pain after treatment with chemotherapeutic agents (25). In that context, drugs that target reactive oxygen and nitrogen species, such as amifostine, are of clinical interest. Amifostine, an inorganic thiophosphate, presents a cytoprotective effect of broad-spectrum selective to healthy tissues (10). The active metabolite of amifostine
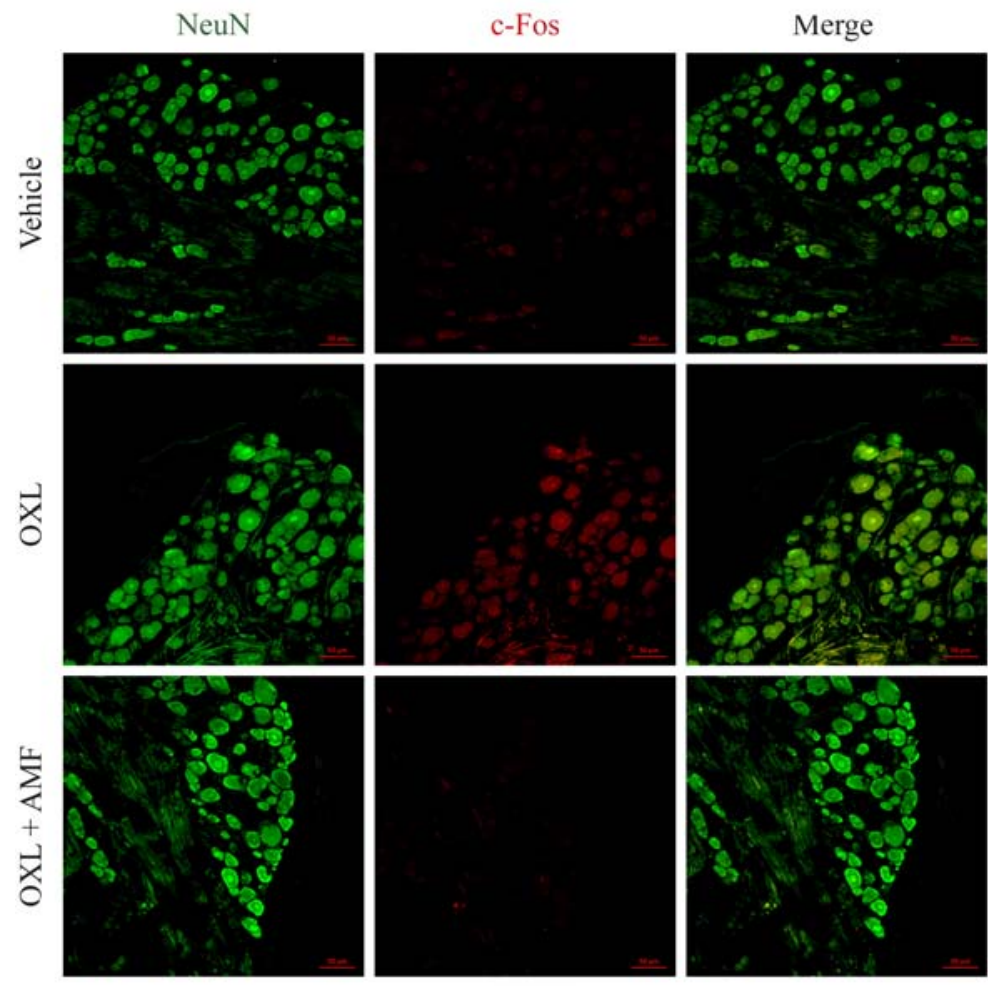

Figure 3. c-Fos expression in the dorsal root ganglia on the $28^{\text {th }}$ experimental day. Green: neuronal marker (NeuN); red: c-Fos; merge: yellow. Magnification $200 \times$; scale bar $50 \mu \mathrm{m}$. OXL: oxaliplatin; AMF: amifostine. 


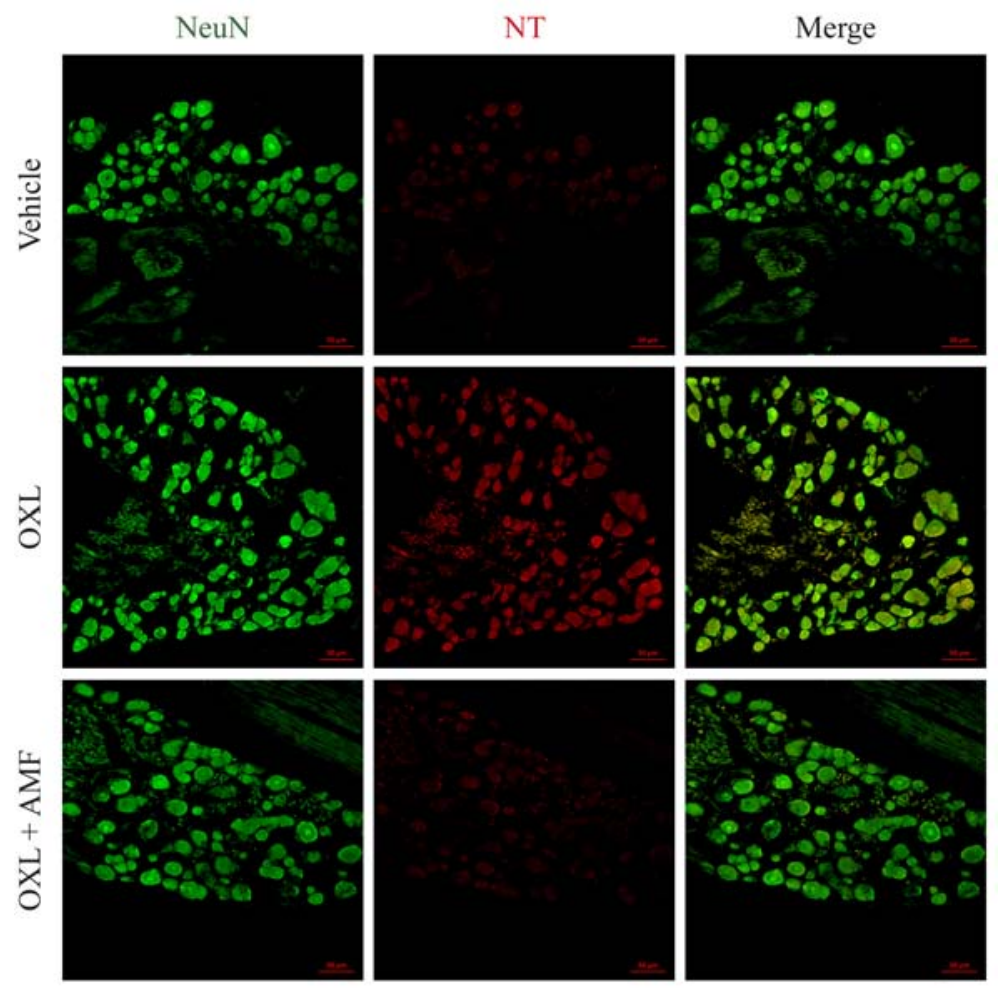

Figure 4. Nitrotyrosine (NT) expression in the dorsal root ganglia on the 28th experimental day. Green: neuronal marker (NeuN); red: nitrotyrosine; merge: yellow. Magnification $200 \times$, scale bar $50 \mu \mathrm{m}$. OXL: oxaliplatin; AMF: amifostine.

(WR-1065) can eliminate free radicals produced in response to chemical substances and ionizing radiation. This metabolite prevents platinum-DNA adducts formed by platinum compounds and promotes the decrease of DNA cross-links caused by $\mathrm{NH}_{2}$ groups of alkylating agents (10). The absence of the alkaline phosphatase enzyme in the tumor possibly explains the selective cytoprotective effect of amifostine on non-tumor cells. This enzyme is required to transform amifostine in its active metabolite. Then, amifostine does not interfere with the antitumor effect of antineoplastic drugs (10).

The neuroprotective effect of amifostine has been demonstrated in the neurotoxicity induced by platinum agents in some clinical trials $(12,28)$. This effect was also confirmed in an in vitro model of oxaliplatin-induced neurotoxicity by using PC12 pheochromocytoma cells (29). Furthermore, amifostine shows a protective effect against 5-fluorouracil-induced hyposalivation, possibly by preventing inflammation (30) and neuronal damage.

Considering that the metabolites of amifostine do not accumulate in the central nervous system due to the blood-brain barrier (10), we decided to study the effect of amifostine on the peripheral nervous system. Oxaliplatin increased the c-Fos expression in neuronal cells in the DRG, as previously demonstrated (21). This increase of
c-Fos expression was also shown in the dorsal horn of the spinal cord (17) and neural tissues following the oxaliplatin injection $(31,32)$. The increased c-Fos expression suggests neuronal activation and neuroplasticity $(22,33)$. Basal levels of c-Fos are usually low, but afferent stimuli during painful conditions may induce its expression $(22,34)$. Interestingly, amifostine prevented c-Fos immunoexpression and neuronal activation caused by the treatment with oxaliplatin, suggesting an inhibitory effect upon nociceptive stimulation.

Azevedo et al. (17) showed that oxaliplatin increases nitrotyrosine expression in the neuronal cells of the spinal cord dorsal horn. In the present study, the increase of nitrotyrosine expression was also found in peripheral nervous system neuronal cells. Nitrotyrosine is formed through the reaction of peroxynitrite $\left(\mathrm{ONOO}^{-}\right)$with the residues of tyrosine amino acids, and is a marker of nitrosative stress (35), potentially contributing to mitochondrial dysfunction (36). In the central and peripheral nervous systems, reactive oxygen species mediate the neurotoxicity consequent to the use of chemotherapeutic agents, such as oxaliplatin $(37,38)$. In this study, we showed that amifostine prevented the increase of nitrotyrosine expression in the DRG of mice treated with oxaliplatin, suggesting the inhibition of nitrosative stress. The preventive effect 


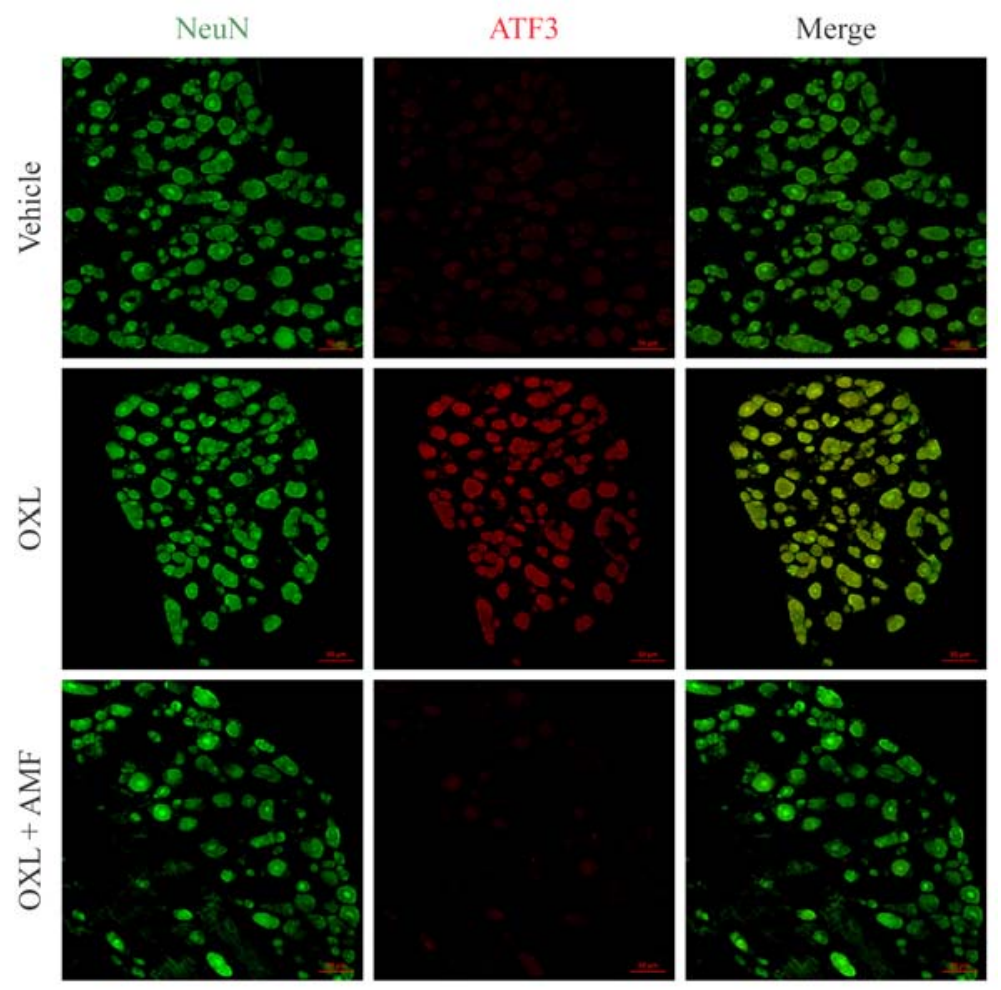

Figure 5. Activating transcription factor 3 (ATF3) expression in the dorsal root ganglia on the 28th experimental day. Green: neuronal marker (NeuN); red: ATF3; merge: yellow. Magnification $200 \times$, scale bar $50 \mu \mathrm{m}$. OXL: oxaliplatin; AMF: amifostine.

of amifostine against nitrosative stress was previously demonstrated in a liver damage model induced by gamma irradiation in rats (39). Other compounds with antioxidant properties, such as the flavonoids rutin and quercetin, have been effective in preventing the nociceptive symptoms and the observed neuronal changes in oxaliplatin-neurotoxicity (17). Thus, antioxidants agents, such as amifostine, are promising compounds for preventing the peripheral sensory neuropathy induced by oxaliplatin $(17,21)$.

Given that amifostine prevented neuronal hyperactivation and nitrosative stress, its effect on the expression of ATF3 was further investigated. ATF3, a marker of neuronal injury, is an activating transcription factor/cyclic AMP response element-binding protein (ATF/CREB) family member expressed by several tissues during stress conditions (23). In the present study, oxaliplatin increased ATF3 expression in neuronal cells of the DRG. The DRG neuronal damage was also shown by Di Cesare Mannelli et al. (40). Moreover, Pereira et al. (21) showed that ATF3 expression remains increased after interruption of oxaliplatin administration, suggesting non-reversible damage. In basal conditions, ATF3 expression is not observed in the DRG and spinal cord neurons (23). Conversely, the expression of this marker increases in neurons submitted to axotomy. Furthermore, it has been shown that ATF3 exerts an essential role in nerve regeneration by increasing the intrinsic growth of injured neurons (24). Notably, amifostine prevented the increase of ATF3 expression in the DRG neurons, showing its protective effect against the neuronal damage caused by oxaliplatin.

In conclusion, amifostine demonstrated a neuroprotective effect on oxaliplatin-induced neurotoxicity. It was confirmed by the prevention of nitrosative stress, neuronal hyperactivation, and damage. This work supports the use of amifostine as a potential strategy for preventing the development of peripheral sensory neuropathy induced by oxaliplatin.

\section{Acknowledgments}

The authors thank Dr. Ronaldo de Albuquerque Ribeiro (in memoriam) for his contribution with the development of this research, Maria Silvandira França Pinheiro (Department of Physiology and Pharmacology, Federal University of Ceará, Brazil) for technical assistance, and the MultiUser Facility of the Drug Research and Development Center of Federal University of Ceará for technical support. This work was funded by the Conselho Nacional de 
Desenvolvimento Científico e Tecnológico (CNPq) and the Fundação Cearense de Apoio ao Desenvolvimento

\section{References}

1. Avan A, Postma TJ, Ceresa C, Avan A, Cavaletti G, Giovannetti $E$, et al. Platinum-induced neurotoxicity and preventive strategies: past, present, and future. Oncologist 2015; 20: 411-432, doi: 10.1634/theoncologist.2014-0044.

2. Hurwitz HI. New agents in colon cancer. Clin Adv Hematol Oncol 2003; 1: 404-405.

3. Goldberg RM, Sargent DJ, Morton RF, Fuchs CS, Ramanathan RK, Williamson SK, et al. A randomized controlled trial of fluorouracil plus leucovorin, irinotecan, and oxaliplatin combinations in patients with previously untreated metastatic colorectal cancer. J Clin Oncol 2004; 22: 23-30, doi: 10.1200/JCO.2004.09.046.

4. Argyriou AA, Bruna J, Marmiroli P, Cavaletti G. Chemotherapy-induced peripheral neurotoxicity (CIPN): an update. Crit Rev Oncol Hematol 2012; 82: 51-77, doi: 10.1016/ j.critrevonc.2011.04.012.

5. Park SB, Lin CS-Y, Krishnan AV, Goldstein D, Friedlander ML, Kiernan MC. Oxaliplatin-induced neurotoxicity: changes in axonal excitability precede development of neuropathy. Brain 2009; 132: 2712-2723, doi: 10.1093/brain/ awp219.

6. Bennedsgaard $K J$, Ventzel $L$, Jensen $A B$, Jensen $A R$, Tankisi H, Finnerup NB. Chronic neuropathic pain following oxaliplatin and docetaxel: a 5-year follow-up questionnaire study. Scand J Pain 2017; 16: 166, doi: 10.1016/j.sjpain. 2017.04.009.

7. Ashley AC, Sargent DJ, Alberts SR, Grothey A, Campbell $\mathrm{ME}$, Morton RF, et al. Updated efficacy and toxicity analysis of irinotecan and oxaliplatin (IROX): intergroup trial N9741 in first-line treatment of metastatic colorectal cancer. Cancer 2007; 110: 670-677, doi: 10.1002/cncr.22831.

8. McWhinney SR, Goldberg RM, McLeod HL. Platinum neurotoxicity pharmacogenetics. Mol Cancer Ther 2009; 8: 10-16, doi: 10.1158/1535-7163.MCT-08-0840.

9. Baron R, Binder A, Wasner G. Neuropathic pain: diagnosis, pathophysiological mechanisms, and treatment. Lancet Neurol 2010; 9: 807-819, doi: 10.1016/S1474-4422 (10)70143-5.

10. Koukourakis MI. Amifostine in clinical oncology: current use and future applications. Anticancer Drugs 2002; 13: 181-209, doi: 10.1097/00001813-200203000-00001.

11. Mercantepe F, Mercantepe T, Topcu A, Y̌ilmaz A, Tumkaya L. Protective effects of amifostine, curcumin, and melatonin against cisplatin-induced acute kidney injury. Naunyn Schmiedebergs Arch Pharmacol 2018; 391: 915-931, doi: 10.1007/s00210-018-1514-4.

12. Lu $P$, Fan $Q X$, Wang LX, Wang $X$, Zong $H$, Wang RL. Prophylactic effect of amifostine on oxaliplatin-related neurotoxicity in patients with digestive tract tumors [in Chinese]. Ai Zheng 2008; 27: 1117-1120.

13. Batista CKLP, Mota JMSC, Souza MLP, Leitão BTA, Souza MHLP, Brito GAC, et al. Amifostine and glutathione prevent ifosfamide- and acrolein-induced hemorrhagic cystitis. Cancer Chemother Pharmacol 2007; 59: 71-77, doi: 10.1007/ s00280-006-0248-z.
Científico e Tecnológico (FUNCAP) (Process PR2-010100054.01.00/15).

14. Bhutia $Y$, Vijayaraghavan $R$, Pathak $U$. Analgesic and antiinflammatory activity of amifostine, DRDE-07, and their analogs, in mice. Indian J Pharmacol 2010; 42: 17-20, doi: 10.4103/0253-7613.62401.

15. Albers JW, Chaudhry V, Cavaletti G, Donehower RC. Interventions for preventing neuropathy caused by cisplatin and related compounds. Cochrane Database Syst Rev 2014: CD005228, doi: 10.1002/14651858.CD005228.pub4

16. Piccolo J, Kolesar JM. Prevention and treatment of chemotherapy-induced peripheral neuropathy. Am J Health Syst Pharm 2014; 71: 19-25, doi: 10.2146/ajhp130126.

17. Azevedo MI, Pereira AF, Nogueira RB, Rolim FE, Brito GA, Wong DV, et al. The antioxidant effects of the flavonoids rutin and quercetin inhibit oxaliplatin-induced chronic painful peripheral neuropathy. Mol Pain 2013; 9: 1-14, doi: 10.1186/ 1744-8069-9-53.

18. Seed TM, Inal CE, Singh VK. Radioprotection of hematopoietic progenitors by low dose amifostine prophylaxis. Int J Radiat Biol 2014; 90: 594-604, doi: 10.3109/09553002. 2014.899450.

19. Cunha TM, Verri Jr WA, Vivancos GG, Moreira IF, Reis S, Parada CA, et al. An electronic pressure-meter nociception paw test for mice. Braz J Med Biol Res 2004; 37: 401-407, doi: 10.1590/S0100-879X2004000300018.

20. Necker R, Hellon RF. Noxious thermal input from the rat tail: modulation by descending inhibitory influences. Pain 1978; 4: 231-242, doi: 10.1016/0304-3959(77)90135-X.

21. Pereira AF, de Oliveira FFB, de Freitas Alves BW, de Menezes KLS, de Mesquita AKV, Lisboa MRP, et al. Neurotoxic effect of oxaliplatin: Comparison with its oxalatefree analogue cis-PtII(1R,2R-DACH)(3-acetoxy-1,1-cyclobutanedicarboxylato). (LLC-1402) in mice. Toxicol Appl Pharmacol 2018; 340: 77-84, doi: 10.1016/j.taap.2018.01.001.

22. Harris JA. Using c-fos as a Neural Marker of Pain. Brain Res Bull 1998; 45: 1-8, doi: 10.1016/S0361-9230(97)00277-3.

23. Tsujino $\mathrm{H}$, Kondo E, Fukuoka T, Dai $\mathrm{Y}$, Tokunaga a, Miki K, et al. Activating transcription factor 3 (ATF3) induction by axotomy in sensory and motoneurons: A novel neuronal marker of nerve injury. Mol Cell Neurosci 2000; 15: 170-182, doi: 10.1006/mcne.1999.0814.

24. Seijffers $R$, Mills $C D$, Woolf CJ. ATF 3 increases the intrinsic growth state of DRG neurons to enhance peripheral nerve regeneration. J Neurosci 2007; 27: 7911-7920, doi: 10.1523/ JNEUROSCI.5313-06.2007.

25. Jaggi AS, Singh N. Mechanisms in cancer-chemotherapeutic drugs-induced peripheral neuropathy. Toxicology 2012; 291: 1-9, doi: 10.1016/j.tox.2011.10.019.

26. Joseph EK, Chen X, Bogen O, Levine JD. Oxaliplatin acts on IB4-positive nociceptors to induce an oxidative stressdependent acute painful peripheral neuropathy. J Pain 2008; 9: 463-472, doi: 10.1016/j.jpain.2008.01.335.

27. Mihara Y, Egashira N, Sada H, Kawashiri T, Ushio S, Yano T, et al. Involvement of spinal NR2B-containing NMDA receptors in oxaliplatin-induced mechanical allodynia in rats. Mol Pain 2011; 7: 1-7, doi: 10.1186/1744-8069-7-8. 
28. De Vos FYF, Bos AME, Schaapveld M, de Swart CAM, de Graaf $\mathrm{H}$, van der Zee AGJ, et al. A randomized phase ॥ study of paclitaxel with carboplatin +/- amifostine as first line treatment in advanced ovarian carcinoma. Gynecol Oncol 2005; 97: 60-67, doi: 10.1016/j.ygyno.2004.11. 052.

29. Ceresa C, Avan A, Giovannetti E, Geldof AA, Avan A, Cavaletti $G$, et al. Characterization of and protection from neurotoxicity induced by oxaliplatin, bortezomib and epothilone-B. Anticancer Res 2014; 34: 517-523.

30. Barbosa SCM, Pereira VBM, Wong DVT, Santana APM, Lucetti LT, Carvalho LL, et al. Amifostine reduces inflammation and protects against 5-fluorouracil-induced oral mucositis and hyposalivation. Braz J Med Biol Res 2019; 52: e8251, doi: 10.1590/1414-431x20188251.

31. Schwingel TE, Klein CP, Nicoletti NF, Dora CL, Hadrich G, Bica CG, et al. Effects of the compounds resveratrol, rutin, quercetin, and quercetin nanoemulsion on oxaliplatininduced hepatotoxicity and neurotoxicity in mice. Naunyn Schmiedebergs Arch Pharmacol 2014; 387: 837-848, doi: 10.1007/s00210-014-0994-0.

32. Toyama S, Shimoyama N, Ishida $\mathrm{Y}$, Koyasu $\mathrm{T}$, Szeto $\mathrm{HH}$, Shimoyama M. Characterization of acute and chronic neuropathies induced by oxaliplatin in mice and differential effects of a novel mitochondria-targeted antioxidant on the neuropathies. Anesthesiology 2014; 120: 459-473, doi: 10.1097/01.anes.0000435634.34709.65.

33. Herdegen T, Zimmermann M. Chapter 17 Immediate early genes (IEGs) encoding for inducible transcription factors (ITFs) and neuropeptides in the nervous system: functional network for long-term plasticity and pain. Prog Brain
Res 1995; 104: 299-321, doi: 10.1016/S0079-6123(08) 61797-5.

34. Kovács KJ. Measurement of immediate-early gene activation- $c$-fos and beyond. J Neuroendocrinol 2008; 20: 665672, doi: 10.1111/j.1365-2826.2008.01734.x.

35. Toblli JE, Cao G, Giani J, Dominici F, Angerosa M. Markers of oxidative/nitrosative stress and inflammation in lung tissue of rats exposed to different intravenous iron compounds. Drug Des Devel Ther 2017; 11: 2251-2263, doi: 10.2147/DDDT.S132612.

36. Areti A, Komirishetty P, Akuthota M, Malik RA, Kumar A. Melatonin prevents mitochondrial dysfunction and promotes neuroprotection by inducing autophagy during oxaliplatinevoked peripheral neuropathy. J Pineal Res 2017; 62, e12393, doi: 10.1111/jpi.12393.

37. Li J, O W, Li W, Jiang ZG, Ghanbari HA. Oxidative stress and neurodegenerative disorders. Int J Mol Sci 2013; 14: 24438-24475, doi: 10.3390/ijms141224438.

38. Di Cesare Mannelli L, Zanardelli M, Failli P, Ghelardini C. Oxaliplatin-induced neuropathy: oxidative stress as pathological mechanism. Protective effect of silibinin. J Pain 2012; 13: 276-284, doi: 10.1016/j.jpain.2011.11.009.

39. Kilciksiz S, Demirel C, Evirgen Ayhan S, Erdal N, Gurgul S, Tamer $\mathrm{L}$, et al. $\mathrm{N}$-acetylcysteine ameliorates nitrosative stress on radiation-inducible damage in rat liver. $J$ BUON 2011; 16: 154-159.

40. Di Cesare Mannelli L, Pacini A, Corti F, Boccella S, Luongo $\mathrm{L}$, Esposito E, et al. Antineuropathic profile of N-palmitoylethanolamine in a rat model of oxaliplatin-induced neurotoxicity. PLoS One 2015; 10: e0128080, doi: 10.1371/ journal.pone. 0128080 . 